\title{
How to save on three universal chemical manufacturing workflows
}

\author{
Mike Jones \\ Waters Corporation, 34 Maple Street, Milford, MA 01757, USA
}

In today's chemical manufacturing industry, time is money. Materials and chemical scientists like you need to verify incoming raw materials, confirm formulated product quality and investigate out of specification (OoS) or contaminated materials. Generating decision-making data more quickly is key, and any reduction in analysis time would be extremely beneficial.

\section{Today's technology doesn't} always meet the mark

Unfortunately, scientists aren't always able to do that with today's technology. Many spectroscopic techniques on the market fail to address common issues like custom ambient ionization techniques tailored for existing mass spectrometry (MS) instrumentation, limiting use to research-based environments. MS instrumentation is also expensive and traditionally challenging to operate, posing additional barriers to adoption.

Atmospheric Pressure Solids Analysis Probe (ASAP) technology and consumables pose additional challenges. Conventional ASAP technology often requires a vertical or near-vertical probe loaded at the top of the instrument, sometimes making it difficult to

DOI: $10.1255 /$ sew. 2021.054
(C) 2021 The Authors
Published under a Creative Commons
BY-NC-ND licence
CC

physically reach the probe. Further challenges include the complex, manual process of cleaning the ASAP consumable, which poses a high risk of contamination to the source surface with the sample material. ASAP MS workflows also require significant knowledge of the underlying MS technology for optimal conditions-a steep learning curve.

\section{A better answer: meet RADIAN ASAP}

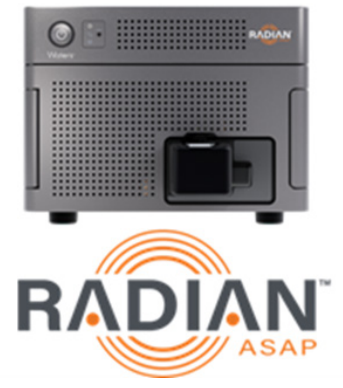

There must be a better way. Now you can quickly, easily and cost-effectively analyze solids, liquids and solutions with the RADIAN ASAP Direct Mass Detector, a novel, dedicated direct analysis system from Waters Corporation.

With RADIAN ASAP, you can quickly and easily analyze with minimal sample preparation. Now, your users can get the mass spectral data they need for rapid, informed decision-making, all without the need for time-consuming chromatographic LC-MS or GC-MS workflows.

With RADIAN ASAP, your lab will get:

- A better fit with typical sampling and analysis workflows over traditional sources with the ASAP source that incorporates a horizontal sample loader
- A mass analyzer based on proven, compact format and single quadrupole technology used in thousands of customer laboratories since 2013-the Waters ACQUITY QDa

- High quality mass spectral data that eliminate the need for timeconsuming, specialist analytical service laboratories

- Hardware with cutting-edge informatics tools like LivelD ${ }^{\mathrm{TM}}$, offering quick results with minimal training and knowledge of MS

The RADIAN ASAP system and informatics solutions provide benefits in three universal workflows for the chemical manufacturing industry: advanced formulation Research \& Development (R\&D), chemical manufacturing process analysis and raw material authenticity/release testing.

\section{An advanced formulation R\&D solution}

Analyzing and monitoring active ingredient/additive chemical profiles is critical in formulation R\&D across industries like automotive, polymers, fast-moving consumer goods (FMCG), oil and gas, and fine and speciality chemicals.

With RADIAN ASAP, analytical scientists or formulation engineers can more easily observe the components of advanced materials and apply a thermal gradient temperature ramp that separates components based on their different boiling points. In Figures 1 and 2, both the polymeric material and the processing additives were easily identified using this approach-speeding up the formulation design decision-making process. 


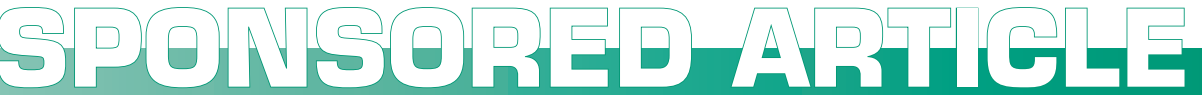

\section{PEG 600 polymer + four additives}

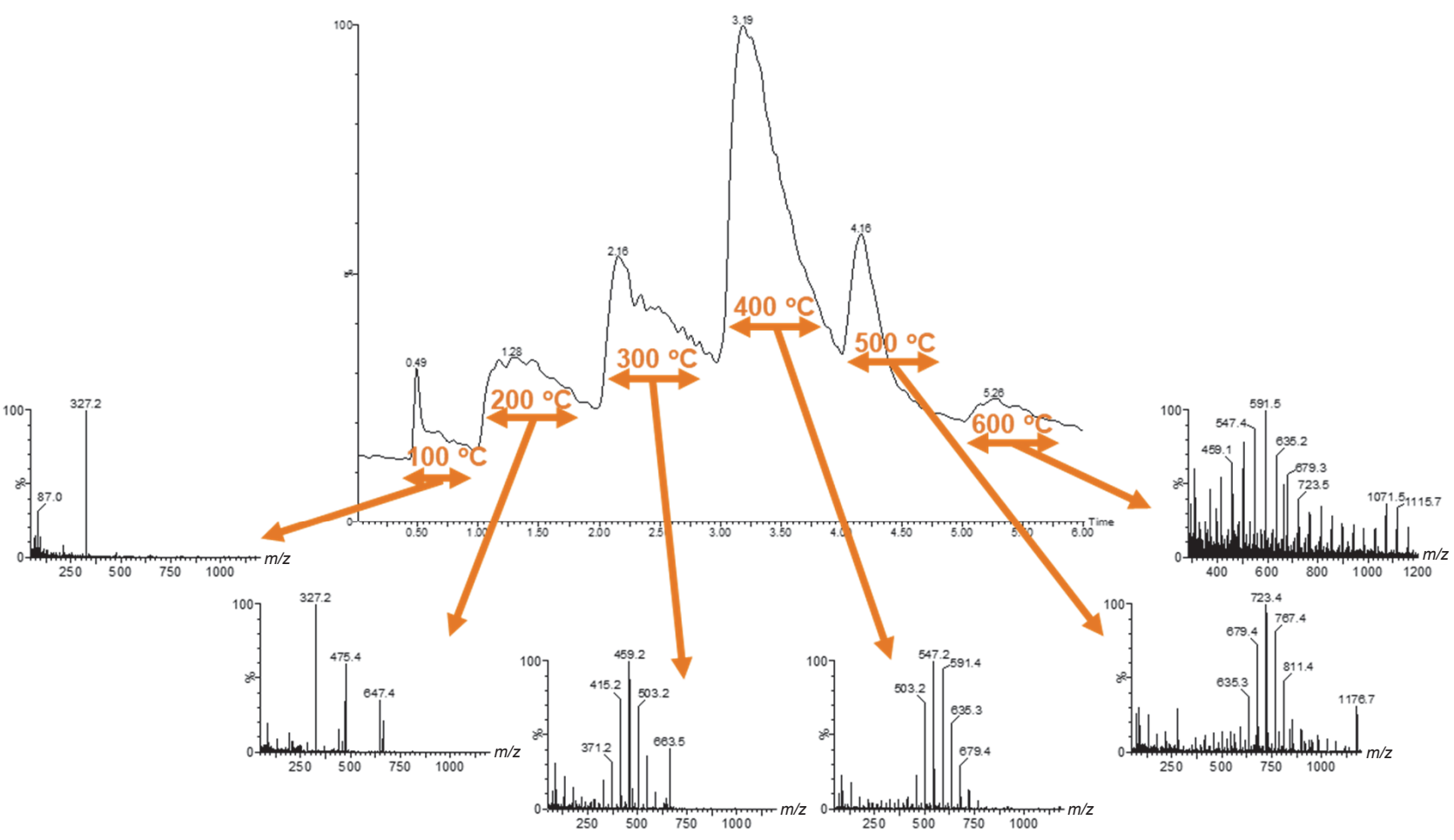

Figure 1. RADIAN ASAP provides rapid analysis of a polymer formulation with minimal sample preparation and the deployment of a thermal gradient to help deconvolute the complex sample.

\section{PEG 600 polymer + four additives}
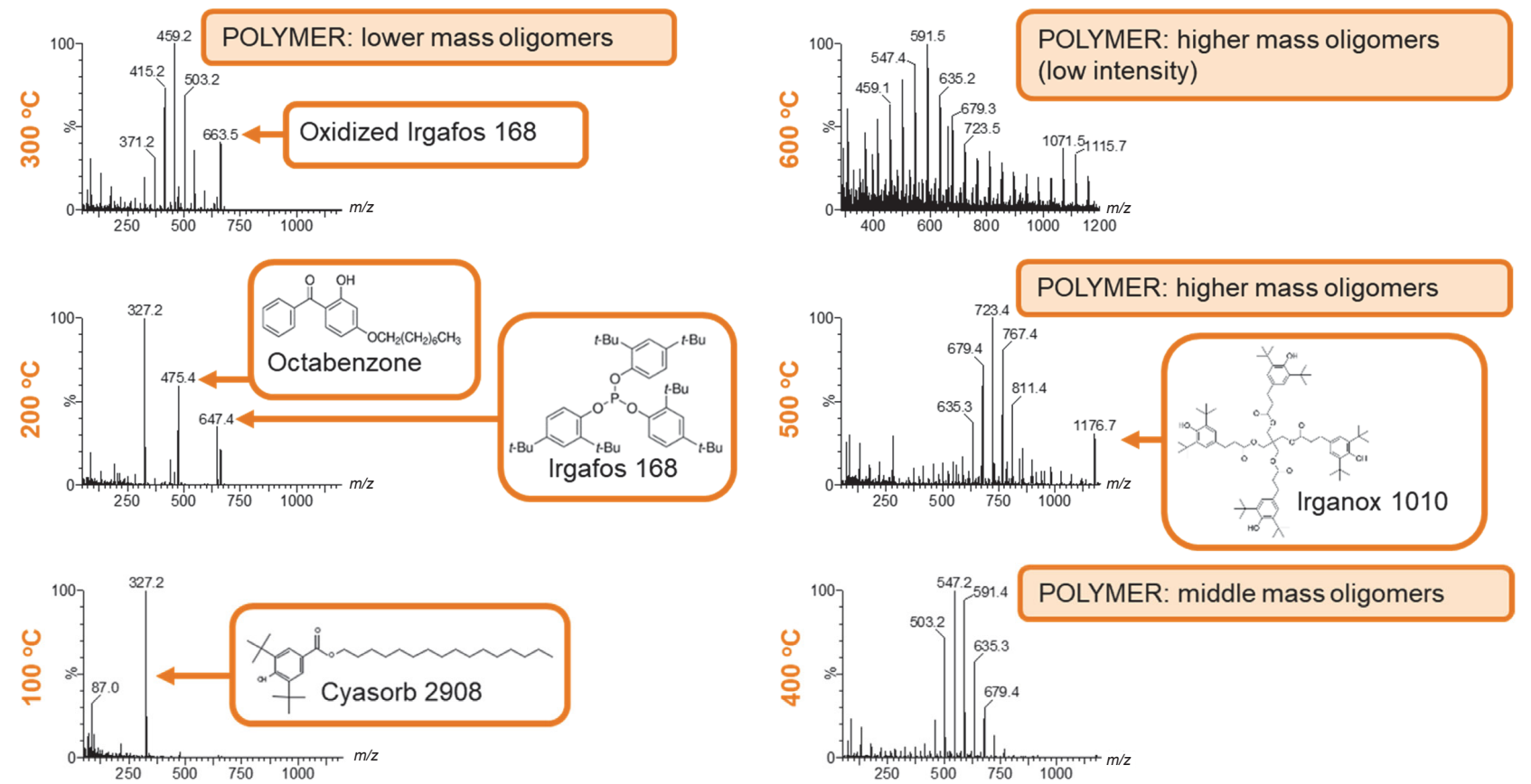

Figure 2. Identification of the additive chemicals and polymeric species based on observed $\mathrm{m} / \mathrm{z}$. 


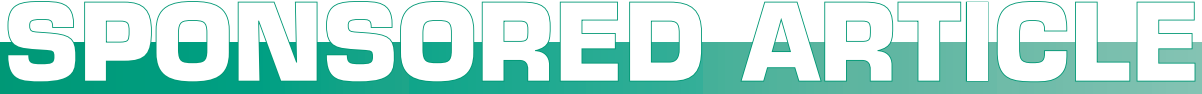

The answer for chemical manufacturing process analysis

With chemical manufacturing process analysis, minimizing the risk of batch failures, and ensuring final product quality and efficacy are critical. With RADIAN ASAP, chemists and chemical engineers can monitor results in real time, so they can:

- Design and control reactions in process vessels, increasing product yields and purity and reducing side reactions and by-products

- Reduce costs and risks associated with unexpected processes
- Increase laboratory and plant efficiency with rapid turnaround in sample analysis for reaction monitoring, speeding decision-making

In Figures 3 and 4, a manufacturing process for a substituted amino propanol is analyzed using the RADIAN ASAP system.

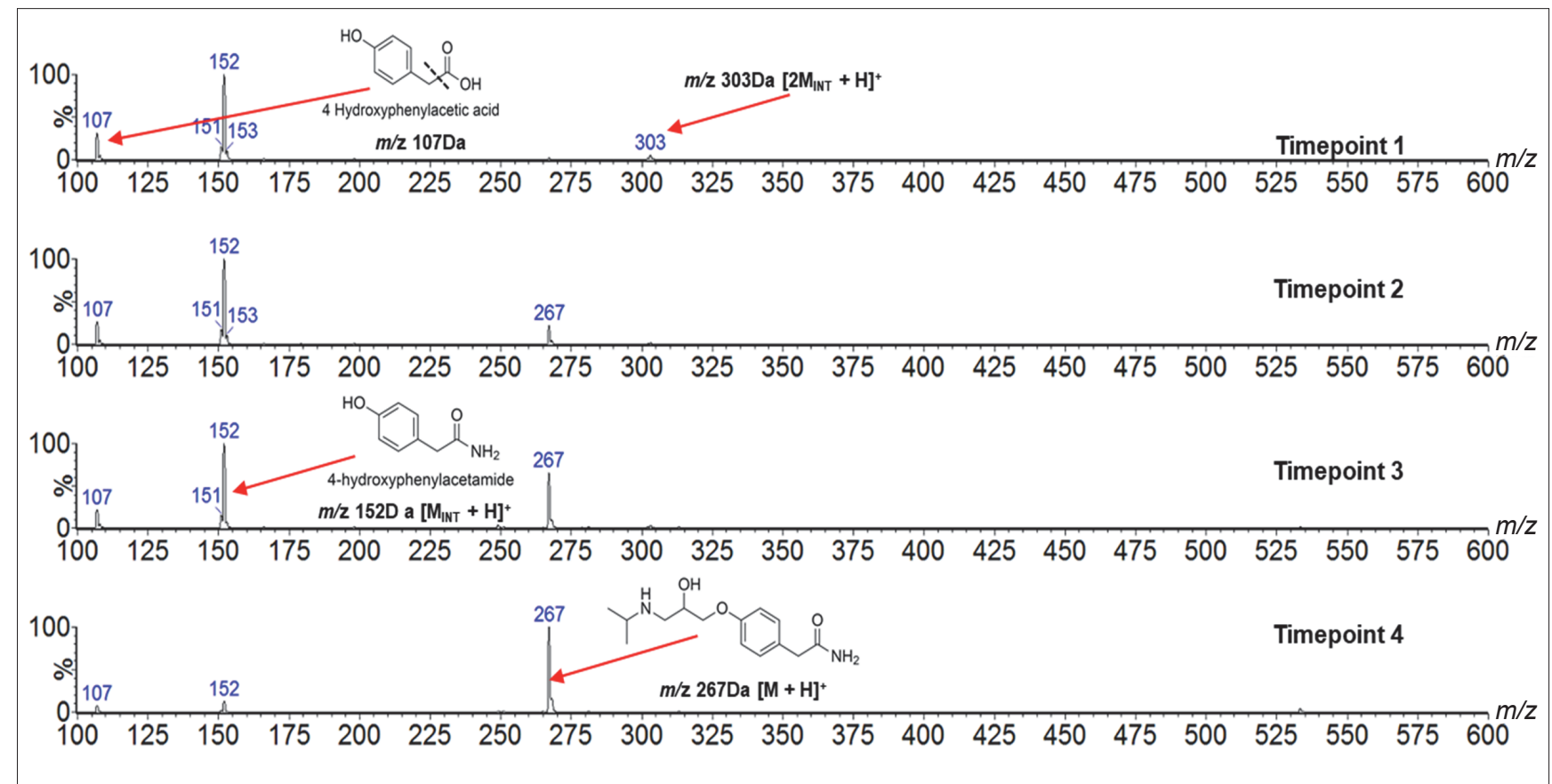

Figure 3. Starting material, 4-hydroxyphenyl acetic acid, has a diminishing response with formation and subsequent diminution of an intermediate, 4-hydroxyphenyl acetamide, increasing presence of reaction product over four time points.

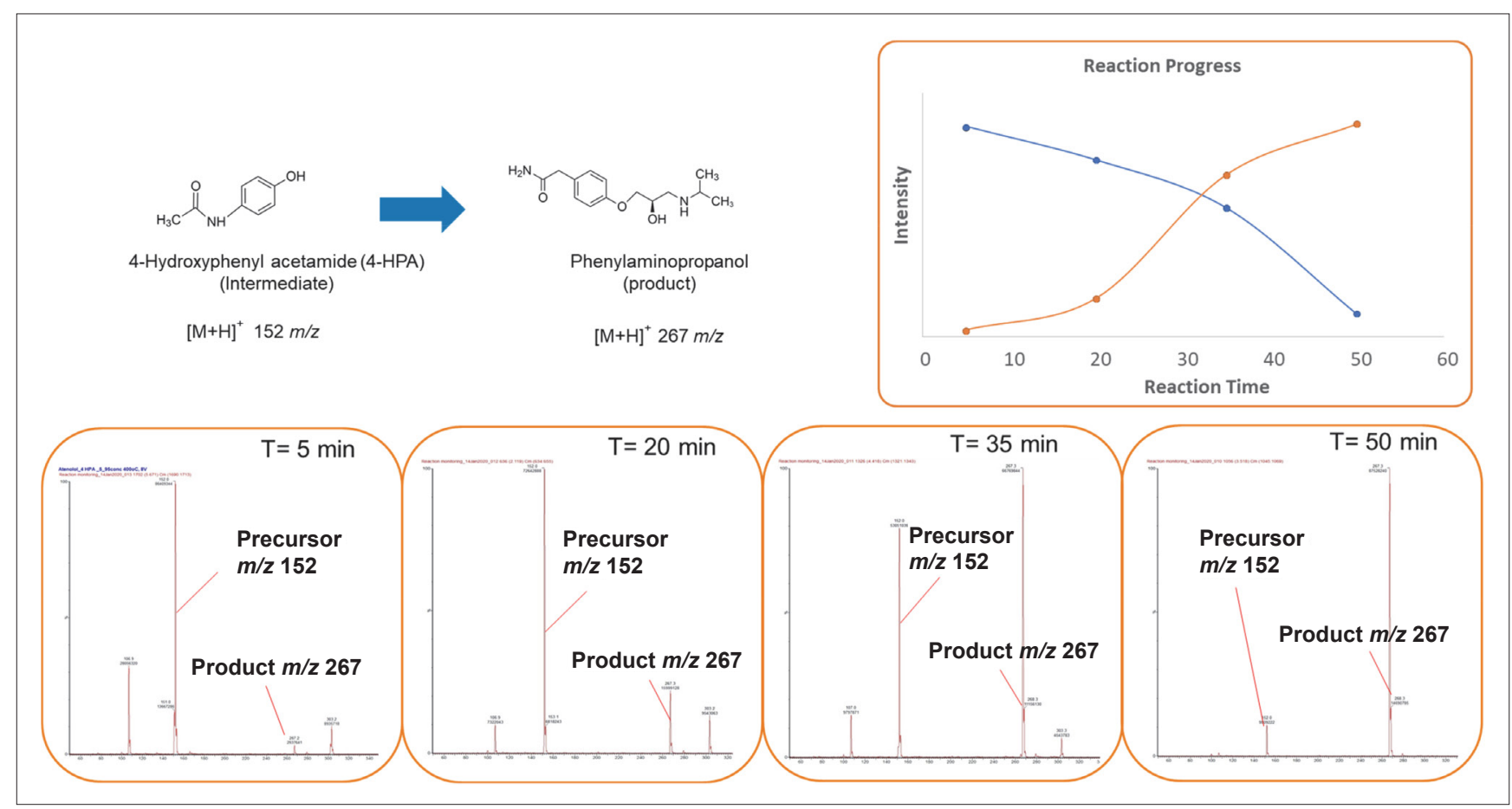

Figure 4. Relative concentrations of reaction intermediate and product, completing the manufacturing process after fifty minutes. 


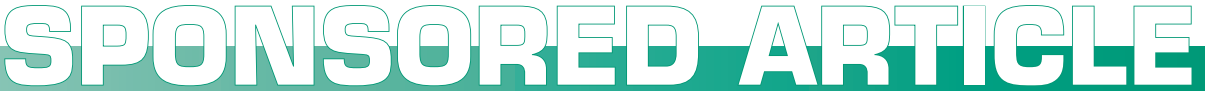
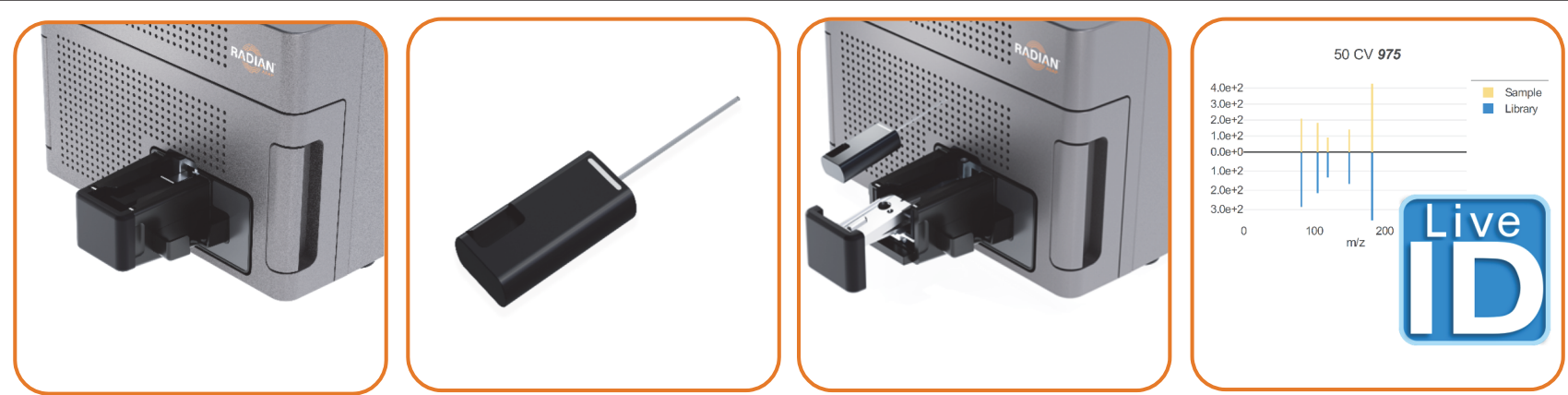

\section{Clean the capillary}

\section{Load sample on capillary}
3. Insert capillary to start acquisition

Ideal for raw material authenticity and release testing

Today's quality control (QC) scientists need to investigate the authenticity, adulteration and quality of ingredients or raw materials. The LivelD software in RADIAN ASAP uses authentic or verified samples to create and validate a statistical model, which QC scientists can then use with test samples to generate live classifications.
QC scientists can also achieve realtime library matching workflows with LivelD. The software matches all compounds in the library against a sample based on mass spectrometric analysis at four distinct cone voltages to generate individual fragmentation profiles and give a score out of 1000with values close to 1000 indicating the compound of interest is likely to be in the sample. The range of software control options with LivelD allows automated mass confirmation reports in electronic and paper formats.

Find out more about knowing now with RADIAN ASAP

Learn how the robust, easily deployable RADIAN ASAP gives your lab a simple workflow that allows non-expert MS users to generate analyses to answers within minutes. Find out more about RADIAN ASAP by visiting our website today.

\section{RADAN}

\section{Ambient lonization for Accelerated Polymer Analysis}

\author{
Watch Now >
}

[ ON-DEMANAND WEBINAR ]

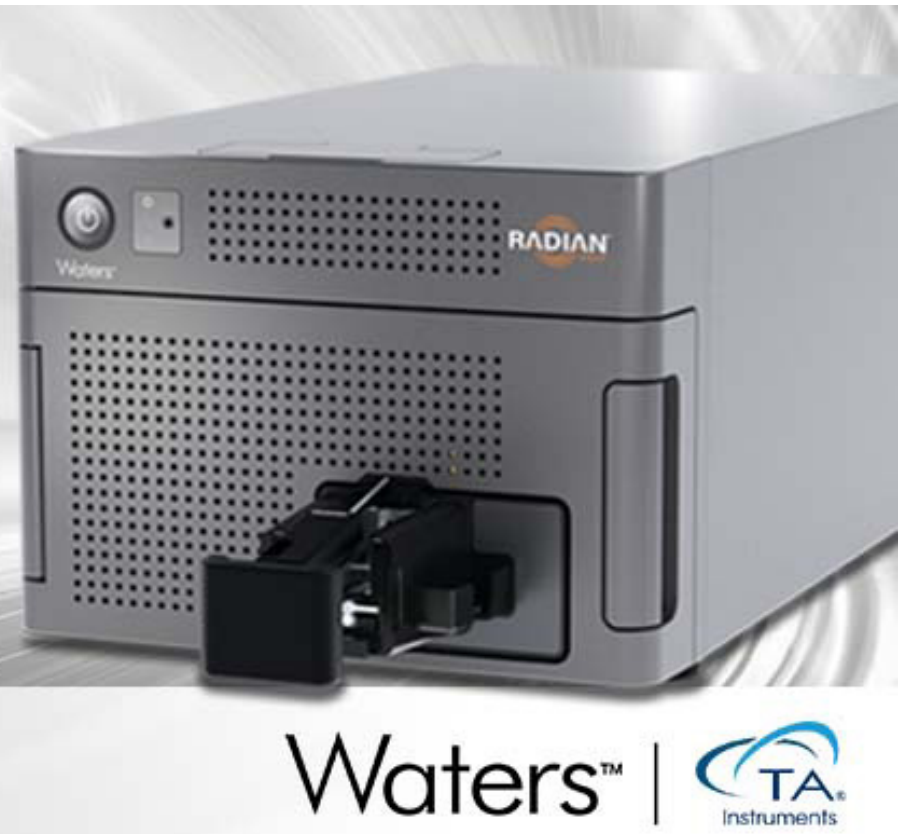

